\title{
Convenient synthesis of naphthopyrans using montmorillonite K-10 as heterogeneous catalyst
}

\author{
RAHMAN HOSSEINZADEH ${ }^{\mathrm{a}, *}$, MARYAM MOHADJERANI ${ }^{\mathrm{b}}$, \\ MOHAMMAD JAVAD ARDESTANIAN ${ }^{\mathrm{a}}$, MOHAMMAD REZA NAIMI-JAMAL ${ }^{\mathrm{c}}$ and \\ ZAHRA LASEMI $^{\mathrm{d}}$ \\ ${ }^{a}$ Faculty of Chemistry, Department of Organic Chemistry, University of Mazandaran, Babolsar, Iran \\ ${ }^{b}$ Department of Molecular and Cell Biology, Faculty of Basic Sciences, University of Mazandaran, \\ Babolsar, Iran \\ ${ }^{c}$ Department of Chemistry, Iran University of Science \& Technology, Naarmak, 16846-11367 Tehran, Iran \\ ${ }^{\mathrm{d}}$ Department of Chemistry, Firoozkooh Branch, Islamic Azad University, Firoozkooh, Iran \\ e-mail: r.hosseinzadeh@umz.ac.ir
}

MS received 23 December 2013; revised 14 March 2014; accepted 9 April 2014

\begin{abstract}
A simple and mild procedure for the synthesis of different naphthopyrans providing high yields in a short reaction time was reported. The reaction of propargylic alcohols with $\alpha$ - or $\beta$-naphthol and dihydroxy naphthalenes in the presence of montmorillonite K-10 was studied. This reaction afforded high yields of the corresponding naphthopyrans. In addition, a number of new phenylene and biphenylen-linked bisnaphthopyrans were synthesized providing excellent yields via the one-pot reaction of bis-propargyl alcohols with $\beta$-naphthol.
\end{abstract}

Keywords. Naphthopyran; propargylic alcohols; naphthol; montmorillonite K-10.

\section{Introduction}

Naphthopyrans, also known as benzochromenes are widely found in nature. ${ }^{1}$ These compounds have been shown to have a wide range of significant biological and pharmacological properties, such as cancer chemopreventive activity, ${ }^{2}$ and anti-viral activity against the hepatitis B virus. ${ }^{3}$ These compounds are also interesting substances due to their photochromic properties. ${ }^{4}$ This photochromic behaviour is the result of a photoinduced reversible opening of the pyran ring that converts the colorless form (the 'closed form') into a highly conjugated coloured form (the 'open form') (scheme 1). Therefore, naphthopyran derivatives have a wide variety of applications such as ophthalmic plastic lenses, solar protective glasses, electronic display systems, optical switches, and temporary or permanent memories. ${ }^{5}$

Several synthetic approaches to naphthopyrans have been reported. These methods include the catalyzed condensation of naphthols with propargylic alcohol by $p$-toluenesulfonic acid in solid phase, ${ }^{6}$ acidic alumina in toluene, ${ }^{7} p$-toluenesulfonic acid in the presence of $(\mathrm{MeO})_{3} \mathrm{CH}$ as a dehydrating agent, ${ }^{8}$ and by indiumtrichloride tetrahydrate under solvent-free ball-milling

*For correspondence conditions. ${ }^{9}$ As in other methods, multistep strategies initiating from chromanones,${ }^{10}$ via Grignard reactions on benzocoumarins followed by dehydration ${ }^{11}$ and reaction of 1-bromonaphthalene-2-ol with 3methylbut-2-enal over organolithium ${ }^{12}$ have been reported. In addition, cycloaddition of naphthol to $\alpha, \beta$ unsaturated aldehydes in refluxing pyridine ${ }^{13}$ or catalyzed by ethylenediamine diacetate ${ }^{14}$ have also been reported. However, there is still a demand for general methods that can efficiently provide naphthopyran rings with various substituents.

The present study reports a very smooth procedure for the synthesis of different naphthopyrans containing phenyl, ferrocenyl and fluorenyl substituents by using montmorillonite $\mathrm{K}-10$ as a suitable catalyst (scheme 2 ).

\section{Experimental}

Melting points were determined with a Stuart Scientific SMP1, or an Electrothermal 9100 apparatus and are uncorrected. Infrared (IR) spectra were recorded with a Bruker Vector 22 FT-IR spectrometer using potassium bromide pellets. $400 \mathrm{MHz}{ }^{1} \mathrm{H}$ NMR and $100 \mathrm{MHz}{ }^{13} \mathrm{C}$ NMR spectra were recorded on a Bruker Avance 400 spectrometer and $500 \mathrm{MHz}{ }^{1} \mathrm{H} \mathrm{NMR}$ and $125 \mathrm{MHz}{ }^{13} \mathrm{C}$ NMR spectra were recorded on a Bruker Avance DRX 


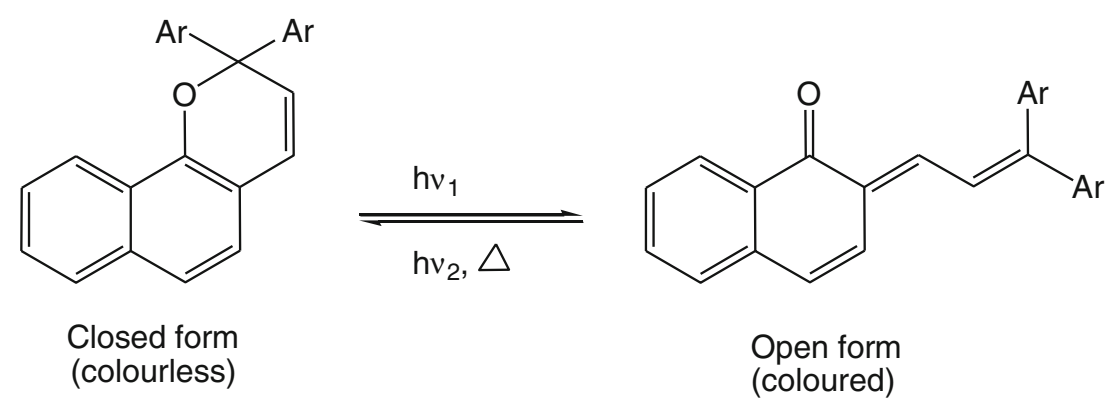

Scheme 1. Photochromic reaction of naphthopyrans.

500 spectrometer. The chemical shifts are reported in ppm ( $\delta$-scale) relative to internal TMS and coupling constants are reported in Hertz $(\mathrm{Hz})$. CHN analyses were done with a CHN Elemental Analyzer LECO 600. The progress of the reactions was checked by thin-layer chromatography (TLC). Silica gel (70-230 mesh) was used for column chromatography.

\subsection{General procedure for the preparation of propargyl alcohols (2)}

To a stirred solution of an acetylenic compound (32 mmol) in dry ether $(50 \mathrm{~mL})$ was gradually added a solution of $1.7 \mathrm{M} n$-BuLi in hexane $(18 \mathrm{~mL}, 30 \mathrm{mmol})$ during $0.5 \mathrm{~h}$ at $-10^{\circ} \mathrm{C}$ under argon. Stirring was continued for $1 \mathrm{~h}$ at the same temperature. The resulting acetylide solution was added dropwise to a stirred solution of a ketone $(28 \mathrm{mmol})$ in dry ether $(100 \mathrm{~mL})$ at $-10^{\circ} \mathrm{C}$ under argon. The reaction mixture was then allowed to warm up to room temperature. After completion of the reaction as monitored by TLC, water $(50 \mathrm{~mL})$ was added and the reaction mixture was extracted with ether $(3 \times 30 \mathrm{~mL})$. The organic layer was dried over anhydrous sodium sulfate. After filtration, the solution was concentrated under reduced pressure and the residue was recrystallized from $n$ hexane/dichloromethane to yield the desired propargyl alcohol (2). 2.1a 1,1,3-Triphenylprop-2-yn-1-ol (2c): Yield: 84\%; M.p. ${ }^{79-81}{ }^{\circ} \mathrm{C} ;{ }^{17}{ }^{1} \mathrm{H}$ NMR $\left(400 \mathrm{MHz}, \mathrm{CDCl}_{3}\right)$ : $\delta$ 7.69-7.72 ( $m, 4 \mathrm{H}), 7.52-7.55(m, 2 \mathrm{H}), 7.28-7.40$ $(m, 9 \mathrm{H}), 2.89(s, 1 \mathrm{H}),{ }^{13} \mathrm{C}$ NMR $\left(100 \mathrm{MHz}, \mathrm{CDCl}_{3}\right)$ : $\delta 145.00,131.79,128.70,128.34,128.33,127.76$, 126.06, 122.41, 91.68, 87.25, 74.85.

2.1b 1-Ferrocenyl-1,3-diphenylprop-2-yn-1-ol (2e): Yield: 78\%; M.p. $86-88^{\circ} \mathrm{C}$; ${ }^{18} \mathrm{IR}(\mathrm{KBr}): 3520,2205$, 1510, 1450, $1300 \mathrm{~cm}^{-1} ;{ }^{1} \mathrm{H}$ NMR (500 MHz, $\left.\mathrm{CDCl}_{3}\right): \delta$ $7.71(d, J=8.4 \mathrm{~Hz}, 2 \mathrm{H}), 7.60($ br $d, J=6.0 \mathrm{~Hz}, 2 \mathrm{H})$, 7.31-7.42 (m, 6H), 4.55 (br s, 1H), $4.43($ br s, $1 \mathrm{H})$, $4.37(s, 5 \mathrm{H}), 4.29(b r s, 1 \mathrm{H}), 4.23(b r s, 1 \mathrm{H}), 3.21(s$, 1H). $\left.{ }^{13} \mathrm{C} \mathrm{NMR} \mathrm{(125} \mathrm{MHz,} \mathrm{CDCl}_{3}\right): \delta 144.50,132.14$, 128.96, 128.85, 128.50, 128.06, 126.03, 123.24, 97.70, $85.41,72.27,69.42,68.97,68.78,65.63$.

2.1c 9-(2-(Trimethylsilyl)ethynyl)-9H-fluoren-9-ol (2f): Yield: $80 \%$; M.p. $120-122^{\circ} \mathrm{C} ;{ }^{19}{ }^{1} \mathrm{H}$ NMR $(400 \mathrm{MHz}$, $\left.\mathrm{CDCl}_{3}\right): \delta 7.69(d, J=7.2 \mathrm{~Hz}, 2 \mathrm{H}), 7.60(d$, $J=7.2 \mathrm{~Hz}, 2 \mathrm{H}), 7.32-7.41(m, 4 \mathrm{H}), 2.50(s$, $1 \mathrm{H}), 0.17(s, 9 \mathrm{H}),{ }^{13} \mathrm{C}$ NMR $\left(100 \mathrm{MHz}, \mathrm{CDCl}_{3}\right)$ : $\delta 147.06,139.19,129.63,128.55,124.33,120.137$, $104.82,88.34,75.05,-0.16$.

2.1d 9-(2-Phenylethynyl)-9H-fluoren-9-ol (2h): Yield: 85\%; Oil; ${ }^{1} \mathrm{H}$ NMR $\left(400 \mathrm{MHz}, \mathrm{CDCl}_{3}\right): \delta 7.82(d, J$ $=8.0 \mathrm{~Hz}, 2 \mathrm{H}) 7.66(d, J=8.0 \mathrm{~Hz}, 2 \mathrm{H}), 7.38-7.49$
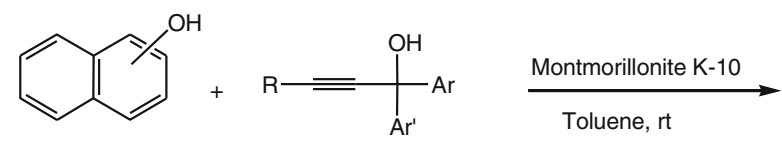

$\mathrm{R}=\mathrm{H}, \mathrm{Ph}, \mathrm{SiMe}_{3}$

$\operatorname{Ar}, \mathrm{Ar}^{\prime}=\mathrm{Ph}, 4-$ Chlorophenyl, Fluorenyl, Ferrocenyl

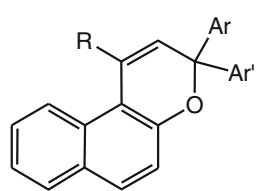<smiles>[Y]C1([Al])C=C(P)c2ccc3ccccc3c2O1</smiles>

Scheme 2. Synthesis of naphthopyrans using propargylic alcohols and naphthols in the presence of montmorillonite $\mathrm{K}-10$ as catalyst. 
$(m, 6 \mathrm{H}), 7.28-7.33(m, 3 \mathrm{H}), 2.94(s, 1 \mathrm{H}) .{ }^{13} \mathrm{C}$ NMR $\left(100 \mathrm{MHz}, \mathrm{CDCl}_{3}\right): \delta 147.23,139.11,132.00,129.74$, $128.66,128.58,128.24,124.49,122.43,120.29,89.10$, 83.19, 75.24 .

2.1e 1,4-Bis(2-bromo-9-hydroxy-9H-fluorene-9-yl)ethynyl-benzen (4): 2 eq 2-Bromofluorenone reacted with 1 eq corresponding acetylide. Yield: 90\%; M.p. 254-256 $6{ }^{\circ} \mathrm{C} ;{ }^{1} \mathrm{H}$ NMR $\left(400 \mathrm{MHz}, \mathrm{CDCl}_{3}\right): \delta=7.87(d$, $J=1.6 \mathrm{~Hz}, 2 \mathrm{H}), 7.74(d d, J=8.0,1.2 \mathrm{~Hz}, 2 \mathrm{H}), 7.60$ $(d, J=7.2 \mathrm{~Hz}, 2 \mathrm{H}), 7.48-7.55(m, 4 \mathrm{H}), 7.35-7.44(m$, $6 \mathrm{H}), 7.26(s, 2 \mathrm{H}), 2.70(s, 2 \mathrm{H}) .{ }^{13} \mathrm{C}$ NMR $(100 \mathrm{MHz}$, $\left.\mathrm{CDCl}_{3}\right): \delta 148.82,146.72,138.08,138.04,132.84$, $131.79,129.99,129.02,127.89,124.42,122.40$, 122.16, 121.61, 120.34, 90.13, 83.12, 74.96. Anal. calcd for $\mathrm{C}_{36} \mathrm{H}_{20} \mathrm{O}_{2} \mathrm{Br}_{2}$ : C, 67.10; $\mathrm{H}, 3.13$; found: $\mathrm{C}$, $66.52 ; \mathrm{H}, 3.05$.

$2.1 \mathrm{f}$ 4,4'-Bis(2-bromo-9-hydroxy-9H-fluorene-9-yl) -ethynyl-biphenyl (5): 2 eq 2-Bromofluorenone reacted with 1 eq coresponding acetylide. Yield: 77\%; M.p. $266-268^{\circ} \mathrm{C} ;{ }^{1} \mathrm{H}$ NMR $\left(400 \mathrm{MHz}\right.$, acetone- $\left.\mathrm{d}_{6}\right): \delta=$ $7.93(d, J=1.2 \mathrm{~Hz}, 2 \mathrm{H}) 7.80(d, J=6.8 \mathrm{~Hz}, 2 \mathrm{H}), 7.75$ $(d, J=8.0 \mathrm{~Hz}, 2 \mathrm{H}), 7.55-7.67(m, 6 \mathrm{H}), 7.42-7.51(\mathrm{~m}$, $10 \mathrm{H}), 2.86(s, 2 \mathrm{H}) .{ }^{13} \mathrm{C}$ NMR $\left(100 \mathrm{MHz}\right.$, acetone- $\left.\mathrm{d}_{6}\right)$ : $\delta=150.72,148.20,140.26,138.60,138.22,132.57$, $132.53,129.81,129.13,127.97,127.10,124.86$, $122.29,122.14,121.57,120.67,91.17,82.36,74.76$. Anal. calcd for $\mathrm{C}_{42} \mathrm{H}_{24} \mathrm{O}_{2} \mathrm{Br}_{2}$ : C, 70.02; H, 3.36; found: C, 69.60; H, 3.28.

\subsection{General procedure for the preparation}

of propargylic alcohols containing terminal acetylenic group $(2, \boldsymbol{R}=\boldsymbol{H})$

Trimethylsilylethynyl alcohols $(23.4 \mathrm{mmol})$ were reacted with $\mathrm{K}_{2} \mathrm{CO}_{3}(19.35 \mathrm{~g}, 140.28 \mathrm{mmol})$ in $\mathrm{MeOH}$ $(90 \mathrm{~mL})$ and THF $(90 \mathrm{~mL})$. After completion of the reaction, the solvent was evaporated and water $(100 \mathrm{~mL})$ and ethyl acetate $(100 \mathrm{~mL})$ were added to the residue. After separation of the organic layer, the solvent was evaporated to give a solid, which was crystallized from $n$-hexane/dichloromethane to obtain the propargylic alcohols 2 .

2.2a 1, 1-Diphenylprop-2-yn-1-ol (2a): Yield: 80\%; Oil ${ }^{20}{ }^{1} \mathrm{H}$ NMR $\left(400 \mathrm{MHz}, \mathrm{CDCl}_{3}\right): \delta$ 7.57-7.61 ( $m, 4 \mathrm{H}), 7.30-7.34(m, 4 \mathrm{H}), 7.23-7.29(m, 2 \mathrm{H}), 3.02$ $(s, 1 \mathrm{H}), 2.87(s, 1 \mathrm{H}),{ }^{13} \mathrm{C}$ NMR $\left(100 \mathrm{MHz}, \mathrm{CDCl}_{3}\right)$ : $\delta=144.43,128.27,127.82,126.01,86.45,75.92$, 75.52 . 2.2b 1-(4-Chlorophenyl)-1-phenylprop-2-yn-1-ol (2b): Yield: 75\%; Oil; ${ }^{20}{ }^{1} \mathrm{H}$ NMR (400 $\left.\mathrm{MHz}, \mathrm{CDCl}_{3}\right): \delta$ 7.60-7.63 ( $m, 2 \mathrm{H}), 7.55-7.58(m, 2 \mathrm{H}), 7.31-7.39(m$, $5 \mathrm{H}), 3.11(s, 1 \mathrm{H}), 2.91(s, 1 \mathrm{H}) .{ }^{13} \mathrm{C}$ NMR $(100 \mathrm{MHz}$, $\left.\mathrm{CDCl}_{3}\right): \delta 144.05,143.05,133.75,128.46,128.44$, $128.12,127.51,125.93,85.96,75.92,73.85$.

2.2c 1-Ferrocenyl-1-phenylprop-2-yn-1-ol (2d): Yield: 78\%; M.p. $90-92^{\circ} \mathrm{C} ;{ }^{18} \mathrm{IR}$ (KBr): 3520, 3250, 2350, 1518, 1350, $1100 \mathrm{~cm}^{-1} ;{ }^{1} \mathrm{H}$ NMR (500 $\left.\mathrm{MHz}, \mathrm{CDCl}_{3}\right)$ : $\delta 7.62$ ((pseudo br s, 2H, ( 7.26-7.32 (m, 3H), $4.48(b r$ $s, 1 \mathrm{H}), 4.18-4.32(m, 8 \mathrm{H}), 3.19(s, 1 \mathrm{H}), 2.81(s, 1 \mathrm{H})$. ${ }^{13} \mathrm{C} \mathrm{NMR}\left(125 \mathrm{MHz}, \mathrm{CDCl}_{3}\right): \delta 143.83,128.10,127.74$, $125.55,96.67,86.87,71.24,69.11,68.55,68.35$, 65.18 .

2.2d 9-Ethynyl-9H-fluoren-9-ol (2g): Yield: 83\%; M.p. $107-109^{\circ} \mathrm{C} ;{ }^{21}{ }^{1} \mathrm{H} \mathrm{NMR}\left(400 \mathrm{MHz}, \mathrm{CDCl}_{3}\right): \delta=$ $7.73(d d, J=8.8,0.8 \mathrm{~Hz}, 2 \mathrm{H}), 7.64(d d, J=8.8$, $0.8 \mathrm{~Hz}, 2 \mathrm{H}), 7.44(d t, J=9.2,1.6 \mathrm{~Hz}, 2 \mathrm{H}), 7.38(d t, J$ $=9.2,1.5 \mathrm{~Hz}, 2 \mathrm{H}), 2.60(s, 1 \mathrm{H}), 2.50(s, 1 \mathrm{H}) .{ }^{13} \mathrm{C} \mathrm{NMR}$ $\left(100 \mathrm{MHz} \mathrm{CDCl}_{3}\right): \delta 146.60,139.13,129.89,128.66$, $124.27,120.28,83.85,74.59,71.41$.

\subsection{General procedure for the preparation of naphthopyrans}

A mixture of a propargylic alcohol $(1.0 \mathrm{mmol}), \beta$ naphthol $(1.0 \mathrm{mmol})$, and montmorillonite K-10 (0.4 g) in toluene $(10 \mathrm{~mL})$ was reacted at room temperature. After the disappearance of the starting materials (monitored by TLC), ethyl acetate $(20 \mathrm{~mL})$ was added and the reaction mixture was filtered. The filtrate was then transferred into a separatory funnel, and washed with water $(50 \mathrm{~mL})$. The organic phase was dried over anhydrous $\mathrm{Na}_{2} \mathrm{SO}_{4}$ and filtered and the solvent was evaporated in a rotary evaporator. The crude product was purified by passing it over a column of silica gel, using a mixture of n-hexane and ethyl acetate as the eluent (4:1). All known products were characterized by comparison of their physical and spectroscopic data with those in literature.

2.3a 3,3-Diphenyl-[3H]-naphtho[2,1-b]pyran (3a): Yield: 97\%; M.p. $161-163^{\circ} \mathrm{C} ;{ }^{6}{ }^{1} \mathrm{H}$ NMR $(400 \mathrm{MHz}$, $\left.\mathrm{CDCl}_{3}\right): \delta=7.98(d, J=8.4 \mathrm{~Hz}, 1 \mathrm{H}), 7.74(d, J=$ $8.0 \mathrm{~Hz}, 1 \mathrm{H}), 7.68(d, J=8.8 \mathrm{~Hz}, 1 \mathrm{H}), 7.47-7.53(\mathrm{~m}$, $5 \mathrm{H}), 7.25-7.36(m, 8 \mathrm{H}), 7.23(d, J=9.2 \mathrm{~Hz}, 1 \mathrm{H}), 6.30$ $(d, J=10.0 \mathrm{~Hz}, 1 \mathrm{H}) .{ }^{13} \mathrm{C}$ NMR $\left(100 \mathrm{MHz}, \mathrm{CDCl}_{3}\right)$ : $\delta 150.56,144.86,129.83,129.35,128.52,128.10$, 
$127.72,127.53,127.02,126.62,123.61,121.33$, $119.54,118.36,114.00,82.55$.

2.3b 3-(4-Chlorophenyl)-3-phenyl-[3H]-naphtho [2,1-b]pyran (3b): Yield: $85 \%$; M.p. $157-159^{\circ} \mathrm{C} ;{ }^{22}$ IR (KBr): 3476, 3386, 2926, 1631, 1451, 812, 759, $717 \mathrm{~cm}^{-1} ;{ }^{1} \mathrm{H}$ NMR $\left(400 \mathrm{MHz}, \mathrm{CDCl}_{3}\right): \delta=7.97(d$, $J=8.4 \mathrm{~Hz}, 1 \mathrm{H}), 7.74(d, J=8.0 \mathrm{~Hz}, 1 \mathrm{H}), 7.69(d$, $J=8.8 \mathrm{~Hz}, 1 \mathrm{H}), 7.43-7.50(\mathrm{~m}, 5 \mathrm{H}), 7.26-7.37(\mathrm{~m}$, $7 \mathrm{H}), 7.20(d, J=8.8 \mathrm{~Hz}, 1 \mathrm{H}), 6.24(d, J=10.0 \mathrm{~Hz}$, 1H). ${ }^{13} \mathrm{C}$ NMR $\left(100 \mathrm{MHz}, \mathrm{CDCl}_{3}\right): \delta 150.34,144.42$, 143.36, 133.47, 130.02, 129.76, 129.40, 128.55,128.52, $128.27,128.22,127.71,127.21,126.90,126.75,123.76$, $121.31,119.96,118.23,114.00,82.09$.

2.3c 1,3,3-Triphenyl-[3H]-naphtho[2,1-b]pyran (3c): Yield: 98\%; M.p. $210-212^{\circ} \mathrm{C} ;{ }^{231} \mathrm{H}$ NMR $(400 \mathrm{MHz}$, $\left.\mathrm{CDCl}_{3}\right): \delta 7.77(d, J=8.0 \mathrm{~Hz}, 1 \mathrm{H}), 7.73(d, J=$ $8.0 \mathrm{~Hz}, 1 \mathrm{H}), 7.62(d, J=8.0 \mathrm{~Hz}, 4 \mathrm{H}), 7.41(t, J$ $=4.5 \mathrm{~Hz}, 6 \mathrm{H}), 7.30-7.36(m, 4 \mathrm{H}), 7.22-7.27(\mathrm{~m}$, $3 \mathrm{H}), 7.16(d, J=6.8 \mathrm{~Hz}, 1 \mathrm{H}), 7.05-7.08(m, 1 \mathrm{H})$, $6.27(s, 1 \mathrm{H}) .{ }^{13} \mathrm{C}$ NMR $\left(100 \mathrm{MHz}, \mathrm{CDCl}_{3}\right): \delta 152.56$, $144.48,141.29,137.18,130.85,130.26,129.80$, 129.40, 128.46, 128.37,128.02, 127.64, 127.49, 127.10, $126.48,125.03,123.14,118.75,116.57,82.12$.

2.3d 3-(Ferrocenyl)-3-phenyl-[3H]-naphtho[2,1-b]pyran (3d): Yield: 95\%; M.p. $145-147^{\circ} \mathrm{C} ;{ }^{4 d}{ }^{1} \mathrm{H}$ NMR $\left(400 \mathrm{MHz}, \mathrm{CDCl}_{3}\right): \delta=7.99(d, J=11.2 \mathrm{~Hz}, 1 \mathrm{H})$, 7.69-7.76 $(m, 2 \mathrm{H}), 7.56(d, J=10.0 \mathrm{~Hz}, 2 \mathrm{H}), 7.36$ $7.49(m, 1 \mathrm{H}), 7.19-7.34(m, 6 \mathrm{H}), 6.43(d, J=13.1 \mathrm{~Hz}$, $1 \mathrm{H}), 4.41(s, 1 \mathrm{H}), 4.26(s, 2 \mathrm{H}), 4.16(s, 6 \mathrm{H}) .{ }^{13} \mathrm{C} \mathrm{NMR}$ $\left(100 \mathrm{MHz}, \mathrm{CDCl}_{3}\right): \delta 151.11,145.35,129.87,129.71$, $129.25,128.56,127.73,127.33,127.08,126.61$, $125.98,123.47,121.25,118.23,113.58,95.22,80.04$, $69.26,68.86,68.28,66.86$.

2.3e 3-(Ferrocenyl)-1,3-diphenyl-[3H]-naphtho[2,1b]pyran (3e): Yield: 70\%; M.p. $193-195^{\circ} \mathrm{C}$; IR (KBr): 3070, 2928, 2859, 1626, 952, 844, $745 \mathrm{~cm}^{-1} ;{ }^{1} \mathrm{H}$ NMR (400 MHz, $\left.\mathrm{CDCl}_{3}\right): \delta 7.68-7.74(m, 5 \mathrm{H}), 7.34$ $7.42(m, 5 \mathrm{H}), 7.23-7.27(m, 2 \mathrm{H}), 7.17-7.20(m, 2 \mathrm{H})$, $6.98-7.07(m, 2 \mathrm{H}), 6.51(s, 1 \mathrm{H}), 4.15-4.23(m, 9 \mathrm{H}) .{ }^{13} \mathrm{C}$ NMR $\left(100 \mathrm{MHz}, \mathrm{CDCl}_{3}\right): \delta 153.06,143.67,141.67$, $136.31,130.62,130.15,129.85,129.08,128.58$, $128.36,128.08,127.56,127.46,127.38,126.55,126.45$, $124.97,123.02,118.72,116.44,95.45,79.53,69.62$, 68.87, 68.80, 67.79, 67.63. Anal. calcd for $\mathrm{C}_{35} \mathrm{H}_{26} \mathrm{FeO}$ : C, 81.08; H, 5.06; found: C, 81.06; H, 5.07.
$2.3 \mathrm{f}$ I'-Trimethylsilyl-spiro[9H-fluorene-9,3'-[3'H]naphtho[2,1-b]pyran] (3f): Yield: 66\%; M.p. 171$173^{\circ} \mathrm{C}$; IR (KBr): $3070,2927,1626,1074,952,844$, 745, $697 \mathrm{~cm}^{-1} ;{ }^{1} \mathrm{H}$ NMR $\left(400 \mathrm{MHz}, \mathrm{CDCl}_{3}\right): \delta 8.22$ $(d, J=8.4 \mathrm{~Hz}, 1 \mathrm{H}), 7.84(d, J=8.0 \mathrm{~Hz}, 1 \mathrm{H}), 7.68$ $(d d, J=10.8,3.6 \mathrm{~Hz}, 3 \mathrm{H}), 7.54(t t, J=8.4,1.4 \mathrm{~Hz}$, $1 \mathrm{H}), 7.38-7.45(\mathrm{~m}, 5 \mathrm{H}), 7.16-7.20(\mathrm{~m}, 3 \mathrm{H}), 6.32$ $(s, 1 \mathrm{H}), 0.34(s, 9 \mathrm{H}),{ }^{13} \mathrm{C}$ NMR $\left(100 \mathrm{MHz}, \mathrm{CDCl}_{3}\right)$ : $\delta$ 152.26, 146.36, 139.69, 137.69, 136.10, 131.08, $130.10,129.79,129.20,128.62,128.31,125.36$, $125.28,125.02,123.63,120.65,120.01,119.25,83.53$, 1.45. Anal. calcd for $\mathrm{C}_{28} \mathrm{H}_{24} \mathrm{SiO}: \mathrm{C}, 82.96 ; \mathrm{H}, 5.97$; found: C, 82.94; H, 5.99.

2.3g Spiro[9H-fluorene-9,3'-[3'H]-naphtho[2,1-b] pyran] (3g): Yield: 70\%; M.p. $200-203^{\circ} \mathrm{C} ;{ }^{6}{ }^{1} \mathrm{H}$ NMR $\left(400 \mathrm{MHz}, \mathrm{CDCl}_{3}\right): \delta 8.11(d, J=8.0 \mathrm{~Hz}, 1 \mathrm{H}), 7.82$ $(d, J=8.0 \mathrm{~Hz}, 1 \mathrm{H}), 7.68-7.70(m, 3 \mathrm{H}), 7.55-7.59$ $(m, 3 \mathrm{H}), 7.48(d, J=12.0 \mathrm{~Hz}, 1 \mathrm{H}), 7.39-7.45(m$, $3 \mathrm{H}), 7.24-7.28(\mathrm{~m}, 2 \mathrm{H}), 7.07(d, J=8.0 \mathrm{~Hz}, 1 \mathrm{H}), 5.74$ $(d, J=10.0 \mathrm{~Hz}, 1 \mathrm{H}) .{ }^{13} \mathrm{C} \mathrm{NMR}\left(100 \mathrm{MHz}, \mathrm{CDCl}_{3}\right)$ : $\delta=152.10,147.49,139.03,129.93,129.82,129.47$, $128.67,128.37,126.74,125.21,124.87,123.66$, $121.16,120.27,120.11,118.40,112.98,85.50$.

2.3h I'-Phenyl-spiro[9H-fluorene-9,3'-[3'H]-naphtho [2,1-b]pyran] (3h): Yield: 75\%; M.p. $243-245^{\circ} \mathrm{C} ;{ }^{9,24}$ IR (KBr): 3053, 2925, 1623, 1447, 1240, 1000, 820, $740 \mathrm{~cm}^{-1} ;{ }^{1} \mathrm{H}$ NMR $\left(400 \mathrm{MHz}, \mathrm{CDCl}_{3}\right): \delta 7.83(d, J$ $=8.0 \mathrm{~Hz}, 1 \mathrm{H}), 7.79(d, J=8.8 \mathrm{~Hz}, 1 \mathrm{H}), 7.70(d, J$ $=6.8 \mathrm{~Hz}, 2 \mathrm{H}), 7.49(d, J=8.0 \mathrm{~Hz}, 2 \mathrm{H}), 7.40-7.44$ $(m, 2 \mathrm{H}), 7.36-7.39(m, 3 \mathrm{H}), 7.33-7.35(m, 2 \mathrm{H}), 7.28-$ $7.32(m, 2 \mathrm{H}), 7.24(d, J=8.0 \mathrm{~Hz}, 1 \mathrm{H}), 7.14-7.20$ $(m, 3 \mathrm{H}), 5.97(s, 1 \mathrm{H}) \cdot{ }^{13} \mathrm{C}$ NMR $\left(100 \mathrm{MHz}, \mathrm{CDCl}_{3}\right)$ : $\delta 154.58,146.39,141.24,139.61,138.22,130.65$, $130.58,129.96,129.79,128.63,128.51,128.32$, $127.90,127.63,126.45,126.14,125.29,125.18$, $123.38,120.08,118.97,116.49,84.48$.

$2.3 \mathrm{i}$ 1,4-Bis(spiro[2-bromo-9H-fluorene-9,3'-[3'H]naphtho[2,1-b] pyran-3-yl]) benzene (6): Yield: 97\%; M.p. $297^{\circ} \mathrm{C}$ (dec); IR (KBr): 3053, 1620, 1450, 1231, $1007,815,744 \mathrm{~cm}^{-1} ;{ }^{1} \mathrm{H} \mathrm{NMR}\left(400 \mathrm{MHz}, \mathrm{CDCl}_{3}\right): \delta=$ $7.84(d, J=8.0 \mathrm{~Hz}, 2 \mathrm{H}), 7.79(d, J=8.8 \mathrm{~Hz}, 2 \mathrm{H}), 7.69$ (br s, 2H), $7.65(d, J=7.6 \mathrm{~Hz}, 2 \mathrm{H}), 7.50-7.56(m, 6 \mathrm{H})$, 7.33-7.42 $(m, 12 \mathrm{H}), 7.21(d, J=8.8 \mathrm{~Hz}, 2 \mathrm{H}), 7.14(t t, J$ $=7.2,1.3 \mathrm{~Hz}, 2 \mathrm{H}), 5.93(s, 2 \mathrm{H}) .{ }^{13} \mathrm{C}$ NMR $(100 \mathrm{MHz}$, $\left.\mathrm{CDCl}_{3}\right): \delta=154.23,148.61,145.84,140.49,138.77$, $138.35,138.10,133.07,131.01,130.67,130.17$, $129.70,128.76,128.69,128.61,128.08,126.48$, $125.33,125.25,125.14,123.57,121.88,121.41,120.17$, 
Table 1. Optimization of catalyst and solvent. ${ }^{\mathrm{a}}$

\begin{tabular}{|c|c|c|c|c|}
\hline Entry & Catalyst & $\begin{array}{l}\text { Amount of } \\
\text { catalyst (g) }\end{array}$ & Solvent & $\begin{array}{l}\text { Yield }(\%) \\
\text { of the } \mathbf{3 a}\end{array}$ \\
\hline 1 & $p$-Toluenesulfonic acid & 0.4 & - & 60 \\
\hline 2 & Amberlyst 15 & 0.4 & - & 76 \\
\hline 3 & Montmorillonite K-10 & 0.4 & - & 80 \\
\hline 4 & Montmorillonite KSF & 0.4 & - & 78 \\
\hline 5 & Silica gel & 0.4 & - & Trace \\
\hline 6 & Alumina & 0.4 & - & Trace \\
\hline 7 & $\left(\mathrm{NH}_{4}\right)_{10} \mathrm{H}_{2}\left(\mathrm{~W}_{2} \mathrm{O}_{7}\right)_{6} \cdot \mathrm{xH}_{2} \mathrm{O}$ & 0.4 & - & Trace \\
\hline 8 & $\mathrm{H}_{3} \mathrm{PW}_{12} \mathrm{O}_{40}$ & 0.4 & - & 30 \\
\hline 9 & Montmorillonite K-10 & 0.4 & DMSO & Trace \\
\hline 10 & Montmorillonite K-10 & 0.4 & THF & Trace \\
\hline 11 & Montmorillonite K-10 & 0.4 & $\mathrm{CH}_{3} \mathrm{CN}$ & 30 \\
\hline 12 & Montmorillonite K-10 & 0.4 & $\mathrm{CH}_{2} \mathrm{Cl}_{2}$ & 70 \\
\hline 13 & Montmorillonite K-10 & 0.4 & $n$-Hexane & 75 \\
\hline 14 & Montmorillonite K-10 & 0.4 & Toluene & 97 \\
\hline 15 & - & - & Toluene & - \\
\hline 16 & Montmorillonite K-10 & 0.2 & Toluene & 85 \\
\hline 17 & Montmorillonite K-10 & 1.2 & Toluene & 98 \\
\hline
\end{tabular}

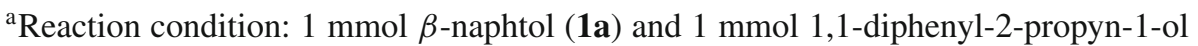
(2a) were reacted at room temperature for $35 \mathrm{~min}$.

118.76, 116.00, 84.27. Anal. calcd for $\mathrm{C}_{56} \mathrm{H}_{32} \mathrm{Br}_{2} \mathrm{O}_{2}: \mathrm{C}$, 75.01; H, 3.60; found: C, 75.03; H, 3.58.

\section{3j 4,4'-Bis(spiro[2-bromo-9H-fluorene-9,3'-[3'H]-} naphtho[2,1-b] pyran-3-yl])biphenyl (7): Yield: 95\%; M.p. $249^{\circ} \mathrm{C}$ (dec); IR (KBr): 3048, 1674, 1621, 1235, 1007, 955, 817, $748 \mathrm{~cm}^{-1}$; ${ }^{1} \mathrm{H}$ NMR $(400 \mathrm{MHz}$, $\left.\mathrm{CDCl}_{3}\right): \delta=7.79-7.84(m, 4 \mathrm{H}), 7.65-7.68(\mathrm{~m}, 7 \mathrm{H}), 7.56$ $(d, J=0.8 \mathrm{~Hz}, 4 \mathrm{H}), 7.32-7.42(m, 12 \mathrm{H}), 7.13-7.23$ $(m, 7 \mathrm{H}), 5.95(s, 2 \mathrm{H})$. Anal. calcd for $\mathrm{C}_{62} \mathrm{H}_{36} \mathrm{Br}_{2} \mathrm{O}_{2}: \mathrm{C}$, 76.55; H, 3.73; found: C, 76.59; H, 3.70.

2.3k 2,2-Diphenyl-[2H]-naphtho[2,1-b]pyran (11): Yield: 60\%; Oil; ${ }^{14}{ }^{1} \mathrm{H}$ NMR $\left(400 \mathrm{MHz}, \mathrm{CDCl}_{3}\right)$ : $\delta 8.38(d, J=8.4 \mathrm{~Hz}, 1 \mathrm{H}), 7.74(d, J=8.4 \mathrm{~Hz}, 1 \mathrm{H})$,
7.53-7.56 ( $m, 4 \mathrm{H}), 7.43-7.51(m, 2 \mathrm{H}), 7.28-7.37(m$, $6 \mathrm{H}), 7.26(t, J=2.4 \mathrm{~Hz}, 1 \mathrm{H}), 7.18(d, J=8.0 \mathrm{~Hz}, 1 \mathrm{H})$, $6.76(d, J=9.6 \mathrm{~Hz}, 1 \mathrm{H}), 6.22(d, J=9.6 \mathrm{~Hz}, 1 \mathrm{H}) .{ }^{13} \mathrm{C}$ NMR (100 MHz, $\left.\mathrm{CDCl}_{3}\right): \delta 147.74,145.20,134.67$, $128.15,127.65,127.47,127.28,126.86,126.29$, $125.56,124.71,124.53,123.86,122.02,120.48$, $115.44,83.19$.

2.31 3,3,9,9-Tetraphenyl-3H,9H-naphtho[2,1-b:6,5b']dipyran (12): Yield: $70 \%$; M.p. $228-230^{\circ} \mathrm{C} ;{ }^{6}{ }^{1} \mathrm{H}$ NMR $\left(400 \mathrm{MHz}, \mathrm{CDCl}_{3}\right): \delta 7.53-7.55(m, 10 \mathrm{H})$, 7.32-7.36 $(m, 8 \mathrm{H}), 7.25-7.29(m, 4 \mathrm{H}), 7.17(d, J$ $=9.6 \mathrm{~Hz}, 2 \mathrm{H}), 7.08(d, J=8.4 \mathrm{~Hz}, 2 \mathrm{H}), 6.10(d$, $J=9.6 \mathrm{~Hz}, 2 \mathrm{H}) .{ }^{13} \mathrm{C} \mathrm{NMR}\left(100 \mathrm{MHz}, \mathrm{CDCl}_{3}\right)$ : $\delta 152.81,144.65,130.96,129.01,128.03,127.53$,<smiles>Oc1ccc2ccccc2c1</smiles>

1

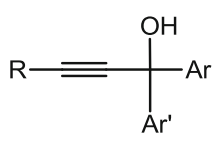

2

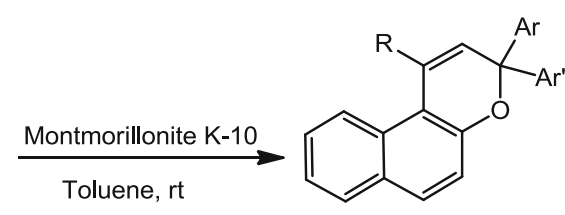

3a-h

\footnotetext{
2a: $A r=A r^{\prime}=P h, R=H$ 2b: $\mathrm{Ar}=\mathrm{Ph}, \mathrm{Ar}=4-$ Chlorophenyl, $\mathrm{R}=\mathrm{H}$ 2c: $A r=A r '=R=P h$ 2d: Ar= Ferrocenyl, $A r^{\prime}=P h, R=H$ 2e: $A r=$ Ferrocenyl, $A r '=R=P h$ 2f: $A r=A r '=$ Fluorenyl, $R=T M S$ 2g: $A r=A r^{\prime}=$ Fluorenyl, $\mathrm{R}=\mathrm{H}$ 2h: $A r=A r '=$ Fluorenyl, $R=P h$
}

Scheme 3. Reaction of propargylic alcohols with $\beta$-naphthol catalyzed by montmorillonite K-10 in toluene at room temperature. 
127.10, 125.71, 124.88, 124.16, 115.90, 114.75, 1000, $957 \mathrm{~cm}^{-1} ;{ }^{1} \mathrm{H}$ NMR $\left(400 \mathrm{MHz}, \mathrm{CDCl}_{3}\right): \delta$ 82.14 .

7.65-7.68 $(m, 8 \mathrm{H}), 7.25-7.33(m, 22 \mathrm{H}), 7.03-7.06$ $(m, 2 \mathrm{H}), 6.73-6.76(m, 2 \mathrm{H}), 6.24(s, 2 \mathrm{H}) .{ }^{13} \mathrm{C} \mathrm{NMR}$ $\left(100 \mathrm{MHz}, \mathrm{CDCl}_{3}\right): \delta 144.59,143.95,141.27,137.01$, $129.38,128.45,128.13,128.05,127.60,127.40$,

$2.3 \mathrm{~m}$ 1,3,3,6,6,8-Hexaphenyl-3H,6H-naphtho[2,1-b:3, 127.03, 126.82, 125.86, 122.30, 118.80, 82.08. Anal. 4-b']dipyran (13): Yield: 98\%; M.p. $250^{\circ} \mathrm{C}$; IR calcd for $\mathrm{C}_{52} \mathrm{H}_{36} \mathrm{O}_{2}$ : C, 90.14; $\mathrm{H}, 5.24$; found: C, 90.15; (KBr): 3049, 1955, 1615, 1398, 1260, 1191, 1116, H, 5.26.

Table 2. Cycloaddition reactions of propargylic alcohols with $\beta$-naphthol (1) catalyzed by montmorillonite K-10.

Entry

${ }^{a}$ Yields refer to isolated products. 
Table 3. Synthesis of some new bis-naphthopyrans by the reaction of bis-acethylenes $\mathbf{4}$ and $\mathbf{5}$ with $\beta$-naphthol catalyzed by montmorillonite K-10.

\begin{tabular}{llll}
\hline Entry & Time $(\mathrm{min}) \quad$ Yield $(\%)^{\mathrm{a}}$ M.p. $\left({ }^{\circ} \mathrm{C}\right)$ \\
297(dec.)
\end{tabular}

${ }^{a}$ Yields refer to isolated products.

\section{Results and discussion}

In a model reaction, $1 \mathrm{mmol}(0.144 \mathrm{~g}) \beta$-naphthol (1a), $1 \mathrm{mmol}$ (0.208 g) 1,1-diphenyl-2-propyn-1-ol (2a), and $0.4 \mathrm{~g}$ of a Lewis acid catalyst were ground together for $10 \mathrm{~min}$ at room temperature. Different Lewis acids such as $p$-toluenesulfonic acid, amberlyst 15 , montmorillonite K-10, montmorillonite KSF, silica gel, alumina, $\left(\mathrm{NH}_{4}\right)_{10} \mathrm{H}_{2}\left(\mathrm{~W}_{2} \mathrm{O}_{7}\right)_{6} \cdot \mathrm{xH}_{2} \mathrm{O}$ and tungstophosphoric acid were investigated. The yields of the products have been summarized in table 1 (entries 1-8). According to these results, montmorillonite $\mathrm{K}-10$ was chosen for this reaction due to its ease of use, availability and low cost and high yield of the product. The model reaction was also repeated in $10 \mathrm{~mL}$ of different solvents as shown in table 1 (entries 9-14). A dramatic increase in the yield of 3a was observed when toluene was taken as reaction media (table 1, entry 14). The amount of the catalyst was also investigated as shown in table 1 (entries 1417). As it is shown, $0.4 \mathrm{~g}$ catalyst for $1.0 \mathrm{mmol}$ of $\mathbf{2 a}$ is sufficient to provide an excellent yield of 3a. The reaction did not take place in the absence of catalyst (table 1 , entry 15).

According to these results, the optimal reaction conditions were chosen as $1.0 \mathrm{mmol}$ of propargylic alcohols, $1.0 \mathrm{mmol}$ of $\beta$-naphthol, $0.4 \mathrm{~g}$ montmorillonite $\mathrm{K}-10$ stirred in toluene at room temperature (scheme 3).

Table 4. Cycloaddition reactions of propargylic alcohols with $\alpha$-naphthol and dihydroxy naphthalens catalyzed by montmorillonite K-10.

Entry Naphthol Propargyl alcohol

${ }^{a}$ Yields refer to isolated products. 
Table 5. Recycling studies of reaction between $\beta$-naphtol (1a) and 1,1-diphenyl-2-propyn-1-ol (2a) in the presence of montmorillonite K-10 to give product $\mathbf{3 a}$.

\begin{tabular}{lccc}
\hline Entry & Cycle & Time (min) & Yield $(\%)^{\mathrm{a}}$ \\
\hline 1 & fresh & 45 & 97 \\
2 & 1 & 45 & 95 \\
3 & 2 & 45 & 92 \\
4 & 3 & 45 & 90 \\
\hline
\end{tabular}

${ }^{\text {a }}$ Yields refer to isolated products.

Under optimized conditions, the substrate scope was subsequently investigated. The results have been listed in table 2. Both terminal and internal alkynes reacted with $\beta$-naphthol smoothly and provided the desired products in excellent yields (table 2, entries 1-3).

It is known that the photochromic properties of the $2 \mathrm{H}$-chromenes can be greatly influenced by the nature of the substituents located in the 2-position. ${ }^{15}$ Previous works have shown that, for example, when a ferrocenyl group replaces a phenyl group in the 2position, the photochromic properties are modified. ${ }^{4 \mathrm{~d}}$ In order to synthesize benzochromenes substituted by the ferrocenyl group in the 2-position, propargylic alcohols having ferrocenyl group (2d and $\mathbf{2 e}$ ) were reacted with $\beta$-naphthol under optimal conditions to yield the corresponding products $\mathbf{3 d}$ and $\mathbf{3 e}$ in substantial yields (table 2, entries 4-5). Propargylic alcohols bearing fluorenyl moieties (2f-h) have also afforded the desired naphthopyran products in good yields (table 2 entries 6-8).

Recently, some interesting results have been obtained on bi-photochromic molecules with two naphthopyrans sharing an aromatic ring linked through the two pyran ring $\mathrm{sp}^{3}-\mathrm{C}$ atoms. These molecules have showed a very significant bathochromic shift of the maximum wavelength of absorption of the open form and high colourabilities due to the extension of $p$-conjugation and the opening of both photochromic systems. ${ }^{16}$ In order to synthesize a number of new bi-photochromic molecules containing conjugated spacers, bis-propargyl alcohols $\mathbf{4}$ and $\mathbf{5}$ were synthesized and reacted with $\beta$ naphthol in the presence of montmorillonite K-10. The yields of new naphtopyrans $\mathbf{6}$ and $\mathbf{7}$ were excellent as tabulated in table 3 .

The reaction of propargylic alcohols with dihydroxynaphthalenes 8-10 also provided high yields of the corresponding naphthopyrans 11-13 (table 4).

Another interesting feature of this method is that the catalyst can be recovered at the end of the reaction and can be used several times without losing its activity. To recover the catalyst, after completion of the reaction, the mixture was filtered and catalyst was washed with EtOH. After activating the catalyst at $100^{\circ} \mathrm{C}$, it was used for further reaction. This process was repeated for three cycles and the yield of product $\mathbf{3 a}$ did not change significantly (table 5).

In order to show the merits and drawbacks of this catalyst, some of our results were compared with other catalysts reported in literature (table 6). As shown in table 6, naphthopyrans $\mathbf{3 a}, \mathbf{3} \mathbf{c}$ and $\mathbf{3 g}$ can be prepared at room temperature and in a short reaction time with excellent yields according to our procedure.

\section{Conclusion}

The present study presents a practical method for the efficient cyclizations of propargylic alcohols with naphthols catalyzed by montmorillonite K-10 in good to high yields. The advantages of this method includes mild reaction conditions, high yields, short reaction times, easy work-up procedures, and ease of recovering and reusing of the catalyst, which make it a useful method for the preparation of naphthopyrans.

Table 6. Comparison of montmorillonite K-10 with other catalysts for the synthesis of $\mathbf{3 a}, \mathbf{3 c}$ and $\mathbf{3} \mathbf{g}$.

\begin{tabular}{|c|c|c|c|c|c|c|}
\hline Entry & Naphthopyran & Catalyst & Reaction Conditions & Time (h) & Yield $(\%)$ & Ref \\
\hline 1 & $3 \mathbf{a}$ & Montmorillonite K-10 & $\mathrm{PhMe} / \mathrm{rt}$ & 0.75 & 97 & - \\
\hline 2 & $\mathbf{3 a}$ & $p$-TsOH/Silica gel & $\mathrm{rt}$ & 1 & 56 & 6 \\
\hline 3 & $\mathbf{3 a}$ & $\mathrm{PPTS} /(\mathrm{MeO})_{3} \mathrm{CH}$ & $\mathrm{ClCH}_{2} \mathrm{CH}_{2} \mathrm{Cl}$ & - & 92 & 8 \\
\hline 4 & $3 c$ & Montmorillonite K-10 & $\mathrm{PhMe} / \mathrm{rt}$ & 0.5 & 98 & - \\
\hline 5 & $3 c$ & $\mathrm{TsOH}$ & Dry $\mathrm{PhMe} / \mathrm{N}_{2} / \mathrm{rt}$ & 2 & 89 & 23 \\
\hline 6 & 3c & Pentafluorophenyl boronic acid /4 A MS & $\mathrm{CH}_{2} \mathrm{Cl}_{2} / \mathrm{rt}$ & 16 & 97 & 24 \\
\hline 7 & 3c & $\mathrm{InCl}_{3} \cdot 4 \mathrm{H}_{2} \mathrm{O}$ & Ball milling at $30 \mathrm{~Hz} / \mathrm{rt}$ & 1 & 97 & 9 \\
\hline 8 & $3 g$ & Montmorillonite K-10 & $\mathrm{PhMe} / \mathrm{rt}$ & 2 & 70 & - \\
\hline 9 & $3 \mathbf{g}$ & $\mathrm{TsOH}$ & Dry $\mathrm{PhMe} / \mathrm{N}_{2} / \mathrm{rt}$ & 2 & 44 & 23 \\
\hline 10 & 3g & $p$-TsOH & Dry $\mathrm{CH}_{2} \mathrm{Cl}_{2} / \mathrm{rt}$ & 2 & 50 & 25 \\
\hline
\end{tabular}




\section{Acknowledgements}

Financial support of this work from the Research Council of University of Mazandaran is gratefully acknowledged.

\section{References}

1. (a) Costa S M O, Lemos T L G, Pessoa O D L, Pessoa C, Montenegro R C and Braz-Filho R $2001 \mathrm{~J}$. Nat. Prod. 64 792; (b) Singh R, Geetanjali G, Chauhan, S M S 2004 Chem. Biodiv. 1 1241; (c) Visentin S, Rolando B, Di Stilo A, Fruttero R, Novara M, Carrbone E, Roussel C, Vanthuyne N and Gasco A 2004 J. Med. Chem. 47 2688

2. Itoigawa $\mathrm{M}$, Ito $\mathrm{C}$, Tan $\mathrm{H} \mathrm{T} \mathrm{W}$, Okuda $\mathrm{M}$, Tokuda $\mathrm{H}$, Nishino H and Furukawa H 2001 Cancer Lett. 174135

3. (a) Kawasaki Y, Goda Y and Yoshihira K 1992 Chem. Pharm. Bull. 40 1504; (b) Marec F, Kollarova I and Jegorov A 2001 Planta. Med. 67 127; (c) Ho L K, Don M J, Chen H C, Yeh S F and Chen J M 1996 J. Nat. Prod. 59 330; d) Chung M I, Jou S J, Cheng H C, Lin C N, Ko F N and Teng C M 1994 J. Nat. Prod. 57313

4. (a) Nakatsuji S 2004 Chem. Soc. Rev. 33 348; (b) Ushakov E N, Nazarov V B, Fedorova O A, Gromov S P, Chebunkova A V, Alfimov M V and Barigelletti F 2003 J. Phys. Org. Chem. 16 306; (c) Hannesschlager P and Brun P 2000 Appl. Organomet. Chem. 14 371; (d) Anguille S, Brun P, Guglielmetti R, Strokach Y P, Ignatin A A, Barachevsky V A and Alfimov M V 2001 J. Chem. Soc., Perkin Trans 2639

5. (a) Van Gemert B 2000 Mol. Cryst. Liq. Cryst. 344 57; (b) Van Gemert, B. 1999 In Organic Photochromic and Thermochromic Compounds J C Crano, R J Guglielmetti (eds.) (New York: Kluwer Academic-Plenum) vol. 1 Chapter 3 pp. 111

6. Tanaka K, Aoki H, Hosomi H and Ohba S 2000 Org. Lett. 22133

7. Gabbut C D, Heron B M and Instone A C 2006 Tetrahedron 62737
8. Zhao W and Carreira E M 2003 Org. Lett. 54153

9. Dong Y W, Wang G W and Wang L 2008 Tetrahedron 6410148

10. Gabbutt C D, Hartley D J, Hepworth J D, Heron B M, Kanjia M and Rahman M 1994 Tetrahedron 502507

11. Cottam J and Livingstone R 1965 J. Chem. Soc. 6646

12. Talley J J 1983 Synthesis 845

13. (a) North J T, Kronenthal D R, Pullockaran A J, Real S D and Chen H Y 1995 J. Org. Chem. 60 3397; (b) Tiabi M and Zamarlik H 1991 Tetrahedron Lett. 32 7251; (c) Calderon-Higginson C, Crombie L, Redshaw S D and Whiting D A 2000 J. Chem. Soc., Perkin. Trans. 1 2491; (d) Lamcharfi E, Menguy L and Zamarlik H 1993 Synth. Commun. 233019

14. Lee Y R and Kim Y M 2007 Helv. Chim. Acta. 902401

15. (a) Van Gemert B, Bergomi $M$ and Knowles D 1994 Mol. Cryst. Liq. Cryst. 246 67; (b) Anguille S, Brun P and Guglielmetti R 1998 Heterocycl Commun. 463

16. (a) Zhao W and Carreira E M 2002 J. Am. Chem. Soc. 124 1582; (b) Zhao W and Carreira E M 2003 U.S. Patent 0078441 A1; (c) Coelho P J, Salvador M A, Heron B M and Carvalho L M 2005 Tetrahedron 6111730

17. Wei L M, Wei L L, Pan W B and Wu M J 2005 Synlett 142219

18. Asgari S 2008 M.Sc. Thesis, University of Mazandaran, Babolsar, Iran

19. Dunn J A, Hunks W J, Ruffolo R, Rigby S S, Brook M A and McGlinchey M J 1999 Organometallics 18 3372

20. Saikachi H and Kitagawa T 1969 Yakugaku Zasshi. 89 1626

21. Shapiro S L, Soloway H and Freedman L 1955 J. Am. Chem. Soc. 774874

22. Guo K and Chen Y 2009 Mol. Cryst. Liqu. Cryst. 50162

23. Pozzo J L, Samat A, Guglielmetti R, Dubest R and Aubard J 1997 Helv. Chim. Acta. 80725

24. McCubbin J A, Nassar C and Krokhin O V 2011 Synthesis 3152

25. Demadrille R, Rabourdin A, Campredon M and Giusti G 2004 J. Photochem. Photobiol. A: Chem. 168143 